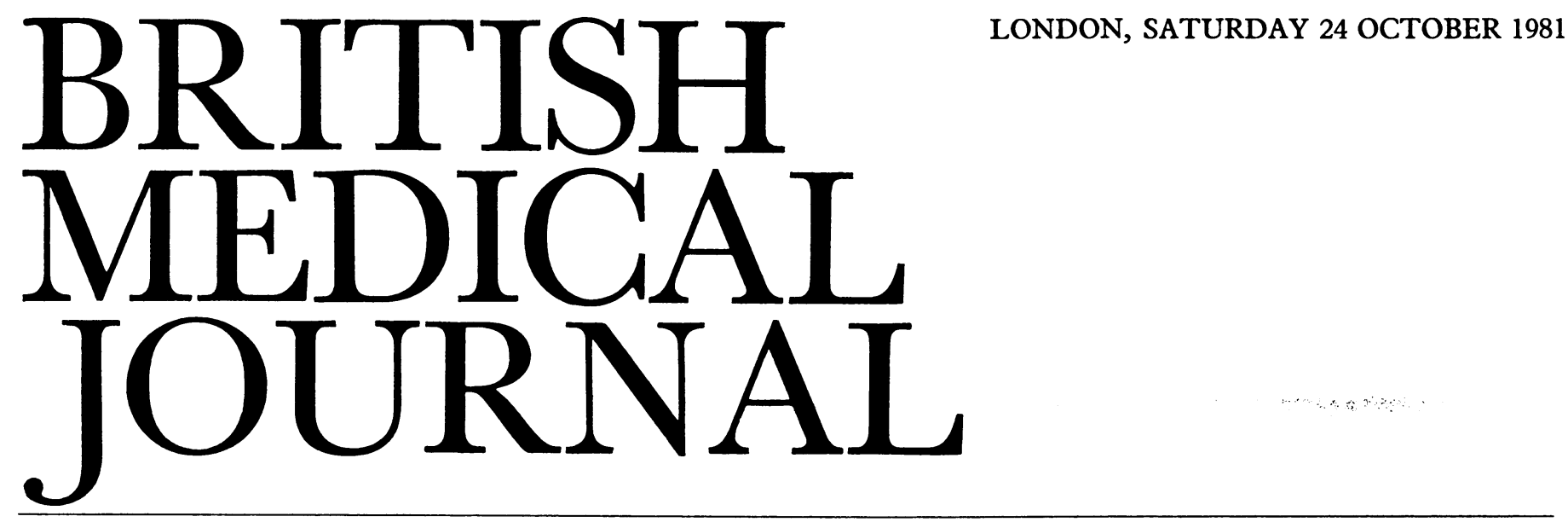

\title{
The progression of coronary artery disease
}

The clinical manifestations of coronary artery disease are rare in youth and common in old age. The underlying disease, however, begins early in life ${ }^{2}$ and is well advanced by the time the patient presents with symptoms of myocardial ischaemia. The silent progression of coronary arterial stenosis in apparently healthy men is a phenomenon about which we know all too little.

The coronary arteries may be visualised by arteriography, a technique introduced by Sones in $1962 .^{3}$ During the past two decades coronary arteriography has proved a safe and invaluable procedure, ${ }^{4}$ being the only reliable method of detecting coronary artery disease, and the best method of predicting the outcome since the prognosis depends on the site and extent of the disease. ${ }^{5}$ The procedure plays such an important part in the management of coronary artery disease that some patients have had it more than once. From these studies we can learn something about the progression of coronary artery disease.

The information has to be interpreted with caution for several reasons, including procedural differences among studies; observer error; the pitfalls of coronary arteriography ${ }^{6}$ and its occasional lack of correlation with necropsy findings ${ }^{7}$; the uncertain part played by coronary vasospasm; and, most important, the bias introduced by studying a selected population. The indications for repeating a coronary arteriogram are varied, but they include a change in the patient's condition, the advent of new forms of treatment, and-for example, in the Netherlands ${ }^{8}$ - a long wait for surgical treatment. Inevitably many patients are excluded by death and this introduces a further bias. Despite these provisos we may draw some conclusions. The first is that adult patients with normal coronary arteriograms seldom, if ever, develop disease. ${ }^{9-11}$ Secondly, about half the patients with coronary artery disease will show progression of disease if arteriography is done two to three years later. ${ }^{81213}$ Thirdly, the correlation between progression and patients' risk factors or clinical state is surprisingly poor. ${ }^{813}$

Two studies have shown a relation between arteriographic evidence of progression and hyperlipidaemia, ${ }^{914}$ and one also between progression and high blood pressure or cigarette smoking. ${ }^{14}$ This latter study may be unreliable because patients who had surgical treatment were included, and bypass is associated with accelerated disease in the proximal segments of grafted vessels. ${ }^{15}$ Two recent careful studies have failed to confirm the relation between risk factors and progression. ${ }^{813}$ In both studies the arteriograms were reviewed by independent investigators and the criteria for diagnosing progression were clearly defined in advance. When the groups whose coronary artery disease progressed were compared with those whose disease remained static there were no significant differences in family history, sex, blood pressure, smoking habits, or serum lipid concentrations. The longer the interval between arteriograms the greater the chance of detecting progression, but the only clinical change that correlated with progression during the interval was myocardial infarction; even so, $27-35 \%$ of those with proved infarction had unchanged coronary arteriograms. Age had little effect; the suggestion that progression was more frequent in younger patients was discounted because they had had longer intervals between their arteriograms. The more severe lesions seen on the first arteriograms seemed more likely to progress, and there was a hint that progression might occur in bouts.

Regression was also seen, but only in $4.7 \%$ of patients. ${ }^{8}$ Coronary artery disease may undoubtedly regress ${ }^{16}$ - and this has been reported in 32 patients so far. ${ }^{17}$ The most convincing evidence comes from a patient whose single severe lesion of the left anterior descending coronary artery regressed, according to the arteriograms, over 10 months; during this time he lost his angina pectoris, the findings of stress electrocardiograp $i y$ and thallium-201 myocardial imaging reverted to normal, and the collateral vessels disappeared. The patient had previously given up smoking, and he adjusted his life style during the 10 months to bring about a fall in his serum lipid concentrations. In another study, on 25 patients who had optimal medical treatment for a year after an episode of unstable angina pectoris, coronary artery disease regressed in five. ${ }^{17}$

So arteriography is teaching us more about the progression and regression of coronary artery disease but we are left with many questions, and at present we can detect a change in the coronary artery anatomy only by repeating the arteriogram. This has important implications. Patients who are being investigated with a view to coronary artery bypass grafting should not have to wait more than a few months for surgical treatment; otherwise they will need a repeat arteriogram because their disease may have progressed and they may require additional or more distal anastomoses. ${ }^{8}$ Patients undergoing medical treatment may show regression of their disease, but because of its unpredictable nature repeat arteriography. is. 
often justifiable. Above all, we should remember that coronary atheroma is an active process that demands active treatment. We tend to overlook this fact because there is no simple, cheap, and painless method of visualising coronary arterial lesions. But just because we cannot see them we must not ignore them.

Consultant Cardiologist,

M C PETCH

Regional Cardiac Unit,

Papworth Hospital,

Cambridge CB3 8RE

${ }^{1}$ Mason JK. Asymptomatic disease of coronary arteries in young men. $\mathrm{Br}$ Med $\mathcal{f} 1963 ; 1: 1234-7$.

${ }^{2}$ Enos WF, Holmes RH, Beyer J. Coronary disease among United States soldiers killed in action in Korea. Preliminary report. $\mathcal{f} A M A 1953 ; 152$ : 1090-3.

${ }^{3}$ Sones FM, Shirley EK. Cine coronary arteriography. Mod Concepts Cardiovasc Dis $1962 ; 31: 735-8$.

${ }^{4}$ Davis K, Kennedy JW, Kemp HG, Judkins MP, Gosselin AJ, Killip T. Complications of coronary arteriography from collaborative study of coronary artery surgery (CASS). Circulation 1979;59:1105-12.

${ }^{5}$ Proudfit WL, Bruschke AV, Sones FM Jr. Natural history of obstructive coronary artery disease: ten-year study of 601 nonsurgical cases. Prog Cardiovasc Dis 1978;21 :53-78.

${ }^{6}$ Gensini GG. Coronary arteriography in heart disease. In: Braunwald E, ed. Heart disease: textbook of cardiovascular medicine. Philadelphia: W B Saunders, 1980:353-60.

7 Isner JM, Kishel J, Kent KM, Ronan JA, Ross AM, Roberts WC. Accuracy of angiographic determination of left main coronary arterial narrowing. Angiographic-histologic correlative analysis in 28 patients. Circulation $1981 ; 63$ :1956-64.

${ }^{8}$ Bruschke AVG, Wijers TS, Kolsters W, Landmann J. The anatomic evolution of coronary artery disease demonstrated by coronary arteriography in 256 nonoperated patients. Circulation 1981 ;63:527-36.

9 Gensini GG, Kelly AE. Incidence and progression of coronary artery disease. Arch Intern Med 1972;129:814-27.

${ }^{10}$ Kimbiris D, Lavine P, van den Boek H, Najmi M, Likoff W. Devolutionary pattern of coronary atherosclerosis in patients with angina pectoris. Coronary arteriographic studies. Am $\mathcal{F}$ Cardiol $1974 ; 33: 7-11$.

11 Marchandise B, Bourassa MG, Chaitman BR, Lesperance J. Angiographic evaluation of the natural history of normal coronary arteries and mild coronary atherosclerosis. Am $\mathcal{F}$ Cardiol $1978 ; 41: 216-20$.

12 Bemis CE, Gorlin R, Kemp HG, Herman MV. Progression of coronary artery disease. A clinical arteriographic study. Circulation 1973;47: 455-64.

${ }^{13}$ Kramer JR, Matsuda Y, Mulligan JC, Aronow M, Proudfit WL. Progression of coronary atherosclerosis. Circulation $1981 ; 63: 519-26$.

${ }^{14}$ Nash DT, Gensini G, Simon H, Arno T, Nash SD. The Erysichthon syndrome. Progression of coronary atherosclerosis and dietary hyperlipidemia. Circulation 1977;56:363-5.

15 Maurer BJ, Oberman A, Holt JH, et al. Changes in grafted and nongrafted coronary arteries following saphenous vein bypass grafting. Circulation $1974 ; 50: 293-300$.

${ }^{16}$ Roth D, Kostuk WJ. Noninvasive and invasive demonstration of spontaneous regression of coronary artery disease. Circulation 1980;62: 888-96.

17 Malinow MR. Regression of atherosclerosis in humans: fact or myth ? Circulation 1981;64:1-3.

${ }^{18}$ Rafflenbeul W, Smith LR, Rogers WJ, Mantle JA, Rackley CE, Russell RO. Quantitative coronary anteriography. Coronary anatomy of patients with unstable angina pectoris re-examined 1 year after optimal medical therapy. Am $\mathcal{F}$ Cardiol $1979 ; 43: 699-707$.

\section{Hepatitis in pregnancy}

A recent paper from Kashmir ${ }^{1}$ has renewed interest in viral hepatitis in pregnancy. This was a prospective study carried out during a water-borne epidemic of proved non-A, non-B hepatitis which showed that its incidence in pregnancy, $17 \%$, was eight times that in non-pregnant women. Hepatitis was more common in the second and third trimesters of pregnancy, developing in $19 \%$ of women compared with only $9 \%$ in the first trimester. Fulminant hepatic failure was also more common in pregnancy-22\% compared with $3 \%$ of men and none of the non-pregnant women with hepatitis. In all, nine patients presented with fulminant hepatic failure in the last trimester and seven died, five with the fetus undelivered. Non-fulminant hepatitis in pregnancy did not, however, cause any increase in maternal or fetal loss.

Both the incidence and severity of hepatitis in pregnancy vary widely around the world. In Western Europe and North America Sever and White ${ }^{2}$ found a low incidence of clinical hepatitis, one in 20000 . In another collective series ${ }^{3}$ a figure of one in 3000 was recorded for North America and Northern Europe, whereas in the Middle East, Africa, and India the reported incidence is around $3 \%$. Findings similar to those from Kashmir have been reported from Iran by Borhanmanesh, ${ }^{4}$ who in a combined prospective and retrospective study of 61 pregnant women found that hepatitis developed much more frequently in the last trimester and that fulminant hepatic failure was also more frequent. Nevertheless, the lack of a standard definition of fulminant hepatic failure means that results from different trials must be interpreted with caution.

The reported effects of hepatitis on the course and outcome of pregnancy show a similar worldwide variation, and there are important differences between the Iranian and Indian studies. In Iran premature delivery or fetal death occurred in half of the cases of non-fulminant hepatitis, and in only one case of the 29 with fulminant hepatic failure did pregnancy continue. In contrast, non-fulminant hepatitis in Kashmir did not have any important influence on the course of pregnancy, and fetal loss in fatal fulminant hepatic failure was as a direct result of the mother's death. In a prospective study in North America Siegel et $a l^{5}$ failed to show an increase in congenital abnormality or fetal mortality after non-fulminant hepatitis. Similar findings have been reported by Hieber et $a l,{ }^{6}$ whose retrospective analysis found no increase in congenital malformation, stillbirth, abortion, or intrauterine malnutrition, though the incidence of prematurity was increased $(27 \%)$. Earlier reports of an increase in Down's syndrome after hepatitis in pregnancy ${ }^{7}$ have not been substantiated in several carefully controlled epidemiological studies. ${ }^{8}$

There is no evidence of transmission of hepatitis to the infant for hepatitis A or for non-A, non-B hepatitis. Vertical transmission does occur with hepatitis $B$, but the frequency varies with racial background. In Taiwan, where up to $20 \%$ of the population are carriers of hepatitis B surface antigen (HBsAg), transmission occurs from mothers without symptoms in nearly $40 \%$ of pregnancies. ${ }^{9}$ Derso et al ${ }^{10}$ have confirmed that babies born to HBsAg-positive mothers of Chinese extraction have an increased risk of hepatitis $\mathrm{B}$ infection compared with babies of HBsAg-positive Europeans. The likelihood of transmission is directly related to the presence of $\mathrm{e}$ antigen in the mother, which correlates closely with virus replication. ${ }^{11}$ The mode of virus transmission remains uncertain, though Beasley and Stevens ${ }^{12}$ have shown that babies born by caesarean section have the same HBsAg carrier rate as those born vaginally, and the finding of $\mathrm{HBsAg}$ in amniotic fluid raises the possibility that infection is acquired from this source.

When hepatitis develops in a pregnant woman (whether it is type $A$, type $B$, or non- $A$, non-B, as in the Indian outbreak) the pregnancy should be allowed to continue to term, provided that placental function is not impaired and hepatic function is stable. Signs of fetal distress, poor progress, or rapid deterioration in maternal condition are all indications for rapid delivery by caesarean section if necessary. If the hepatitis becomes fulminant prompt delivery by caesarean section is recommended to improve the chances of the mother's recovering. Deterioration in the maternal condition may, however, continue after delivery in some cases of fulminant hepatic failure 